\title{
The Relationship Between Precision-Recall and ROC Curves
}

\section{Jesse Davis Mark Goadrich}

Department of Computer Sciences and Department of Biostatistics and Medical Informatics, University of Wisconsin-Madison, 1210 West Dayton Street, Madison, WI, 53706 USA

\section{Introduction}

In machine learning, current research has shifted away from simply presenting accuracy results when performing an empirical validation of new algorithms. This is especially true when evaluating algorithms that output probabilities of class values. Provost et al. (1998) have argued that simply using accuracy results can be misleading. They recommended when evaluating binary decision problems to use Receiver Operator Characteristic (ROC) curves, which show how the number of correctly classified positive examples varies with the number of incorrectly classified negative examples. However, ROC curves can present an overly optimistic view of an algorithm's performance if there is a large skew

\footnotetext{
Appearing in Proceedings of the $23^{\text {rd }}$ International Conference on Machine Learning, Pittsburgh, PA, 2006. Copyright 2006 by the author(s)/owner(s).
}

in the class distribution. Drummond and Holte (2000; 2004) have recommended using cost curves to address this issue. Cost curves are an excellent alternative to ROC curves, but discussing them is beyond the scope of this paper.

Precision-Recall (PR) curves, often used in Information Retrieval (Manning \& Schutze, 1999; Raghavan et al., 1989), have been cited as an alternative to ROC curves for tasks with a large skew in the class distribution (Bockhorst \& Craven, 2005; Bunescu et al., 2004; Davis et al., 2005; Goadrich et al., 2004; Kok \& Domingos, 2005; Singla \& Domingos, 2005). An important difference between ROC space and PR space is the visual representation of the curves. Looking at PR curves can expose differences between algorithms that are not apparent in ROC space. Sample ROC curves and PR curves are shown in Figures 1(a) and 1(b) respectively. These curves, taken from the same learned models on a highly-skewed cancer detection dataset, highlight the visual difference between these spaces (Davis et al., 2005). The goal in ROC space is to be in the upper-left-hand corner, and when one looks at the ROC curves in Figure 1(a) they appear to be fairly close to optimal. In PR space the goal is to be in the upper-right-hand corner, and the PR curves in Figure 1(b) show that there is still vast room for improvement.

The performances of the algorithms appear to be comparable in ROC space, however, in PR space we can see that Algorithm 2 has a clear advantage over Algorithm 1 . This difference exists because in this domain the number of negative examples greatly exceeds the number of positives examples. Consequently, a large change in the number of false positives can lead to a small change in the false positive rate used in ROC analysis. Precision, on the other hand, by comparing false positives to true positives rather than true negatives, captures the effect of the large number of negative examples on the algorithm's performance. Section 2 defines Precision and Recall for the reader unfamiliar with these terms.

We believe it is important to study the connection be- 


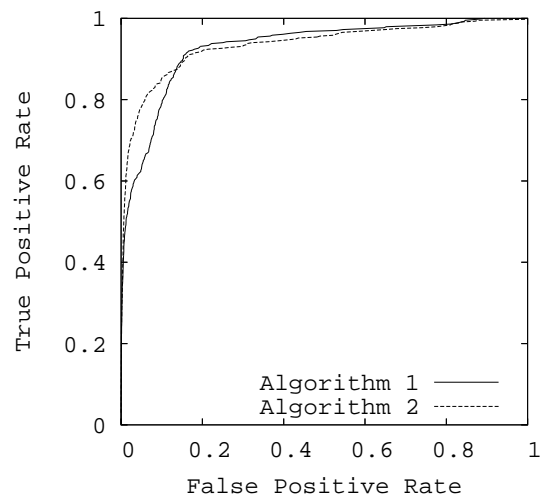

(a) Comparison in ROC space

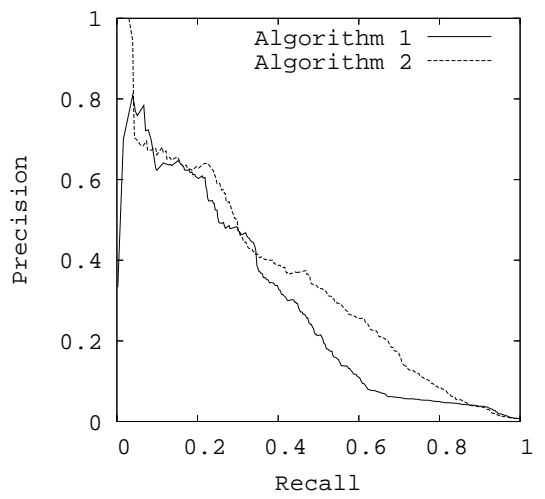

(b) Comparison in PR space

Figure 1. The difference between comparing algorithms in ROC vs PR space

tween these two spaces, and whether some of the interesting properties of ROC space also hold for $\mathrm{PR}$ space. We show that for any dataset, and hence a fixed number of positive and negative examples, the ROC curve and PR curve for a given algorithm contain the "same points." Therefore the PR curves for Algorithm I and Algorithm II in Figure 1(b) are, in a sense that we formally define, equivalent to the ROC curves for Algorithm I and Algorithm II, respectively in Figure 1(a). Based on this equivalence for ROC and $\mathrm{PR}$ curves, we show that a curve dominates in ROC space if and only if it dominates in PR space. Second, we introduce the PR space analog to the convex hull in ROC space, which we call the achievable PR curve. We show that due to the equivalence of these two spaces we can efficiently compute the achievable PR curve. Third we demonstrate that in PR space it is insufficient to linearly interpolate between points. Finally, we show that an algorithm that optimizes the area under the ROC curve is not guaranteed to optimize the area under the PR curve.

\section{Review of ROC and Precision-Recall}

In a binary decision problem, a classifier labels examples as either positive or negative. The decision made by the classifier can be represented in a structure known as a confusion matrix or contingency table. The confusion matrix has four categories: True positives (TP) are examples correctly labeled as positives. False positives (FP) refer to negative examples incorrectly labeled as positive. True negatives (TN) correspond to negatives correctly labeled as negative. Finally, false negatives (FN) refer to positive examples incorrectly labeled as negative.
A confusion matrix is shown in Figure 2(a). The confusion matrix can be used to construct a point in either ROC space or PR space. Given the confusion matrix, we are able to define the metrics used in each space as in Figure 2(b). In ROC space, one plots the False Positive Rate (FPR) on the $x$-axis and the True Positive Rate (TPR) on the $y$-axis. The FPR measures the fraction of negative examples that are misclassified as positive. The TPR measures the fraction of positive examples that are correctly labeled. In PR space, one plots Recall on the $x$-axis and Precision on the $y$-axis. Recall is the same as TPR, whereas Precision measures that fraction of examples classified as positive that are truly positive. Figure 2(b) gives the definitions for each metric. We will treat the metrics as functions that act on the underlying confusion matrix which defines a point in either ROC space or $\mathrm{PR}$ space. Thus, given a confusion matrix $A, \operatorname{RECALL(A)}$ returns the Recall associated with $A$.

\section{Relationship between ROC Space and PR Space}

ROC and PR curves are typically generated to evaluate the performance of a machine learning algorithm on a given dataset. Each dataset contains a fixed number of positive and negative examples. We show here that there exists a deep relationship between ROC and PR spaces.

Theorem 3.1. For a given dataset of positive and negative examples, there exists a one-to-one correspondence between a curve in ROC space and a curve in $P R$ space, such that the curves contain exactly the same confusion matrices, if Recall $\neq 0$. 


\begin{tabular}{c|c|c|} 
& $\begin{array}{c}\text { actual } \\
\text { positive }\end{array}$ & $\begin{array}{c}\text { actual } \\
\text { negative }\end{array}$ \\
\hline predicted positive & $T P$ & $F P$ \\
\hline predicted negative & $F N$ & $T N$ \\
\hline
\end{tabular}

(a) Confusion Matrix

$\begin{array}{ll}\text { Recall } & =\frac{T P}{T P+F N} \\ \text { Precision } & =\frac{T P}{T P+F P} \\ \text { True Positive Rate } & =\frac{T P}{T P+F N} \\ \text { False Positive Rate } & =\frac{F P}{F P+T N}\end{array}$

(b) Definitions of metrics

Figure 2. Common machine learning evaluation metrics

Proof. Note that a point in ROC space defines a unique confusion matrix when the dataset is fixed. Since in PR space we ignore $T N$, one might worry that each point may correspond to multiple confusion matrices. However, with a fixed number of positive and negative examples, given the other three entries in a matrix, $T N$ is uniquely determined. If Recall = 0 , we are unable to recover $F P$, and thus cannot find a unique confusion matrix.

Consequently, we have a one-to-one mapping between confusion matrices and points in PR space. This implies that we also have a one-to-one mapping between points (each defined by a confusion matrix) in ROC space and PR space; hence, we can translate a curve in ROC space to PR space and vice-versa.

One important definition we need for our next theorem is the notion that one curve dominates another curve, "meaning that all other...curves are beneath it or equal to it (Provost et al., 1998)."

Theorem 3.2. For a fixed number of positive and negative examples, one curve dominates a second curve in $R O C$ space if and only if the first dominates the second in Precision-Recall space.

\section{Proof.}

Claim $1(\Rightarrow)$ : If a curve dominates in ROC space then it dominates in PR space. Proof by contradiction. Suppose we have curve I and curve II (as shown in Figure 3) such that curve I dominates in ROC space, yet, once we translate these curves in PR space, curve I no longer dominates. Since curve I does not dominate in PR space, there exists some point $A$ on curve II such that the point $B$ on curve I with identical Recall has lower Precision. In other words, PRECISION $(A)>P R E C I S I O N(B)$ yet $\operatorname{RECALL}(A)=\operatorname{RECALL}(B)$. Since $\operatorname{RECALL}(A)=\operatorname{RECALL}(B)$ and Recall is identical to $T P R$, we have that $T P R(A)=T P R(B)$. Since curve I dominates curve II in ROC space
$F P R(A) \geq F P R(B)$. Remember that total positives and total negatives are fixed and since $\operatorname{TPR}(A)=\operatorname{TPR}(B)$ :

$$
\begin{aligned}
& T P R(A)=\frac{T P_{A}}{\text { Total Positives }} \\
& T P R(B)=\frac{T P_{B}}{\text { Total Positives }}
\end{aligned}
$$

we now have $T P_{A}=T P_{B}$ and thus denote both as $T P$. Remember that $F P R(A) \geq F P R(B)$ and

$$
\begin{aligned}
& F P R(A)=\frac{F P_{A}}{\text { Total Negatives }} \\
& F P R(B)=\frac{F P_{B}}{\text { Total Negatives }}
\end{aligned}
$$

This implies that $F P_{A} \geq F P_{B}$ because

$$
\begin{aligned}
& \operatorname{PRECISION}(A)=\frac{T P}{F P_{A}+T P} \\
& \operatorname{PRECISION}(B)=\frac{T P}{F P_{B}+T P}
\end{aligned}
$$

we now have that $\operatorname{PRECISION}(A) \leq$ $P R E C I S I O N(B)$. But this contradicts our original assumption that $\operatorname{PRECISION~}(A)>$ PRECISION $(B)$.

Claim $2(\Leftarrow)$ : If a curve dominates in $\mathbf{P R}$ space then it dominates in ROC space. Proof by contradiction. Suppose we have curve I and curve II (as shown in Figure 4) such that curve I dominates curve II in PR space, but once translated in ROC space curve I no longer dominates. Since curve I does not dominate in ROC space, there exists some point $A$ on curve II such that point $B$ on curve I with identical $T P R$ yet $F P R(A)<T P R(B)$. Since $R E C A L L$ and $T P R$ are the same, we get that $R E C A L L(A)=R E C A L L(B)$. Because curve I dominates in PR space we know that $P R E C I S I O N(A) \leq P R E C I S I O N(B)$. Remember 


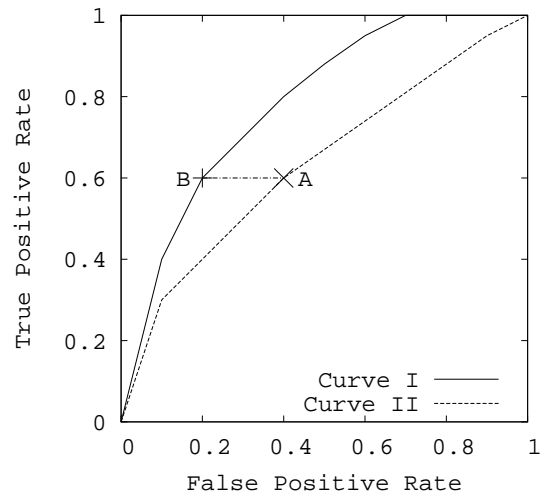

(a) Case 1: $F P R(A)>F P R(B)$

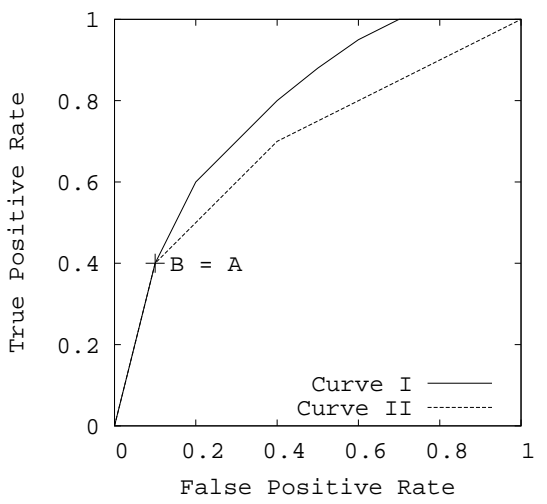

(b) Case 2: $F P R(A)=F P R(B)$

Figure 3. Two cases for Claim 1 of Theorem 3.2

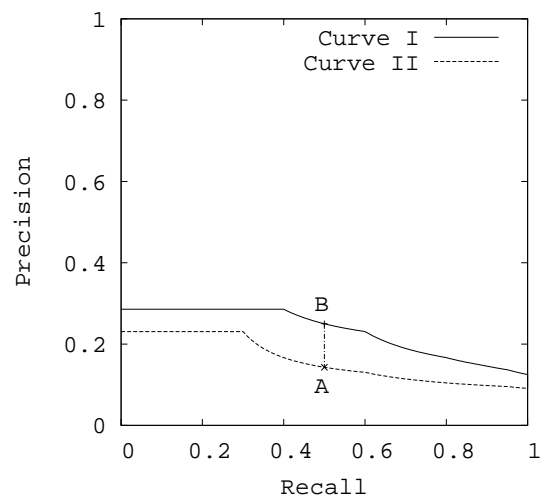

(a) Case 1: PRECISION $(A)<$
PRECISION $(B)$

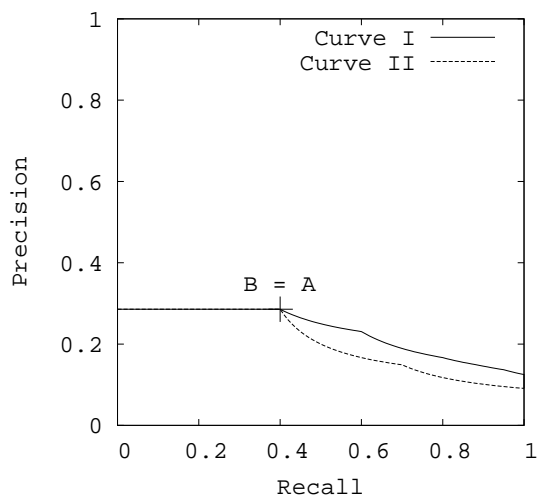

(b) Case 2: PRECISION $(A)=$ $P R E C I S I O N(B)$

Figure 4. Two cases of Claim 2 of Theorem 3.2

that $R E C A L L(A)=R E C A L L(B)$ and

$$
\begin{aligned}
\operatorname{RECALL}(A) & =\frac{T P_{A}}{\text { Total Positives }} \\
\operatorname{RECALL}(B) & =\frac{T P_{B}}{\text { Total Positives }}
\end{aligned}
$$

We know that $T P_{A}=T P_{B}$, so we will now denote them simply as TP. Because PRECISION $(A) \leq$ $\operatorname{PRECISION}(B)$ and

$$
\begin{aligned}
& \operatorname{PRECISION}(A)=\frac{T P}{T P+F P_{A}} \\
& \operatorname{PRECISION}(B)=\frac{T P}{T P+F P_{B}}
\end{aligned}
$$

we find that $F P_{A} \geq F P_{B}$. Now we have

$$
\begin{aligned}
& F P R(A)=\frac{F P_{A}}{\text { Total Negatives }} \\
& F P R(B)=\frac{F P_{B}}{\text { Total Negatives }}
\end{aligned}
$$

This implies that $F P R(A) \geq F P R(B)$ and this contradicts our original assumption that $F P R(A)<$ $F P R(B)$.

In ROC space the convex hull is a crucial idea. Given a set of points in ROC space, the convex hull must meet the following three criteria:

1. Linear interpolation is used between adjacent points.

2. No point lies above the final curve. 


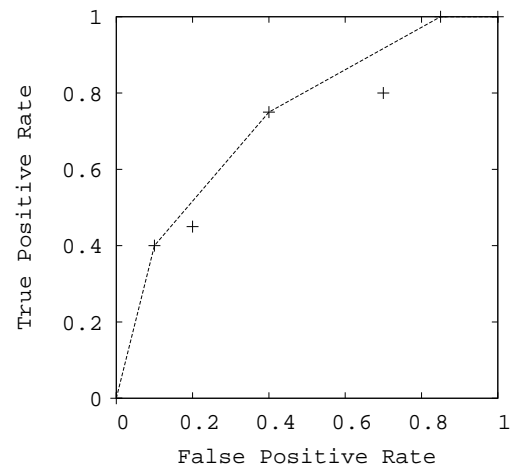

(a) Convex hull in ROC space

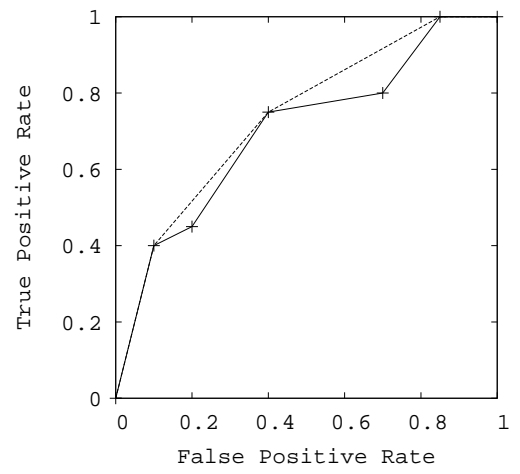

(b) Curves in ROC space

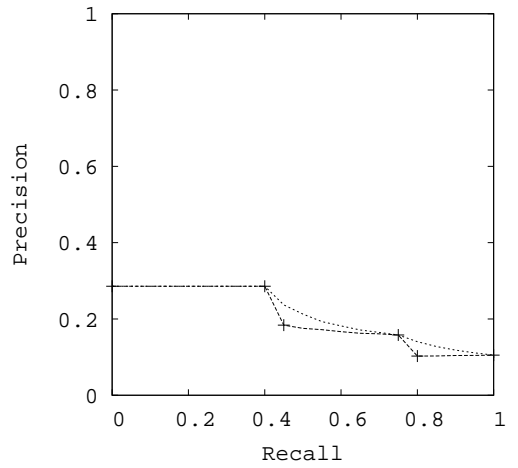

(c) Equivalent curves in PR space

Figure 5. Convex hull and its PR analog dominate the naïve method for curve construction in each space. Note that this achievable PR curve is not a true convex hull due to non-linear interpolation. Linear interpolation in PR space is typically not achievable.

3. For any pair of points used to construct the curve, the line segment connecting them is equal to or below the curve.

Figure 5(a) shows an example of a convex hull in ROC space. For a detailed algorithm of how to efficiently construct the convex hull, see Cormen et al. (1990).

In PR space, there exists an analogous curve to the convex hull in ROC space, which we call the achievable PR curve, although it cannot be achieved by linear interpolation. The issue of dominance in ROC space is directly related to this convex hull analog.

Corollary 3.1. Given a set of points in PR space, there exists an achievable $P R$ curve that dominates the other valid curves that could be constructed with these points.

Proof. First, convert the points into ROC space (Theorem 3.1), and construct the convex hull of these points in ROC space. By definition, the convex hull dominates all other curves that could be constructed with those points when using linear interpolation between the points. Thus converting the points of the ROC convex hull back into PR space will yield a curve that dominates in PR space as shown in Figures 5(b) and 5(c). This follows from Theorem 3.2. The achievable PR curve will exclude exactly those points beneath the convex hull in ROC space.

The convex hull in ROC space is the best legal curve that can be constructed from a set of given ROC points. Many researchers, ourselves included, argue that PR curves are preferable when presented with highly-skewed datasets. Therefore it is surprising that we can find the achievable PR curve (the best legal $\mathrm{PR}$ curve) by first computing the convex hull in ROC space and the converting that curve into PR space. Thus the best curve in one space gives you the best curve in the other space.

An important methodological issue must be addressed when building a convex hull in ROC space or an achievable curve in $\mathrm{PR}$ space. When constructing a ROC curve (or PR curve) from an algorithm that outputs a probability, the following approach is usually taken: first find the probability that each test set example is positive, next sort this list and then traverse the sorted list in ascending order. To simplify the discussion, let class $(i)$ refer to the true classification of the example at position $i$ in the array and $\operatorname{prob}(i)$ refer to the probability that the example at position $i$ is positive. For each $i$ such that $\operatorname{class}(i) \neq \operatorname{class}(i+1)$ and $\operatorname{prob}(i)<\operatorname{prob}(i+1)$, create a classifier by calling every example $j$ such that $j \geq i+1$ positive and all other examples negative.

Thus each point in ROC space or PR space represents a specific classifier, with a threshold for calling an example positive. Building the convex hull can be seen as constructing a new classifier, as one picks the best points. Therefore it would be methodologically incorrect to construct a convex hull or achievable PR curve by looking at performance on the test data and then constructing a convex hull. To combat this problem, the convex hull must be constructed using a tuning set as follows: First, use the method described above to find a candidate set of thresholds on the tuning data. Then, build a convex hull over the tuning data. Finally use the thresholds selected on the tuning data, when 
building an ROC or PR curve for the test data. While this test-data curve is not guaranteed to be a convex hull, it preserves the split between training data and testing data.

\section{Interpolation and AUC}

A key practical issue to address is how to interpolate between points in each space. It is straightforward to interpolate between points in ROC space by simply drawing a straight line connecting the two points. One can achieve any level of performance on this line by flipping a weighted coin to decide between the classifiers that the two end points represent.

However, in Precision-Recall space, interpolation is more complicated. As the level of Recall varies, the Precision does not necessarily change linearly due to the fact that $F P$ replaces $F N$ in the denominator of the Precision metric. In these cases, linear interpolation is a mistake that yields an overly-optimistic estimate of performance. Corollary 3.1 shows how to find the achievable PR curve by simply converting the analogous ROC convex hull; this yields the correct interpolation in PR space. However, a curve consists of infinitely many points, and thus we need a practical, approximate method for translation. We expand here on the method proposed by Goadrich et al. (2004) to approximate the interpolation between two points in PR space.

Remember that any point $A$ in a Precision-Recall space is generated from the underlying true positive $\left(T P_{A}\right)$ and false positive $\left(F P_{A}\right)$ counts. Suppose we have two points, $A$ and $B$ which are far apart in Precision-Recall space. To find some intermediate values, we must interpolate between their counts $T P_{A}$ and $T P_{B}$, and $F P_{A}$ and $F P_{B}$. We find out how many negative examples it takes to equal one positive, or the local skew, defined by $\frac{F P_{B}-F P_{A}}{T P_{B}-T P_{A}}$. Now we can create new points $T P_{A}+x$ for all integer values of $\mathrm{x}$ such that $1 \leq x \leq T P_{B}-T P_{A}$, i.e. $T P_{A}+1, T P_{A}+2, \ldots, T P_{B}-1$, and calculate corresponding FP by linearly increasing the false positives for each new point by the local skew. Our resulting intermediate Precision-Recall points will be

$$
\left(\frac{T P_{A}+x}{\text { Total Pos }}, \frac{T P_{A}+x}{T P_{A}+x+F P_{A}+\frac{F P_{B}-F P_{A}}{T P_{B}-T P_{A}} x}\right) .
$$

For example, suppose we have a dataset with 20 positive examples and 2000 negative examples. Let $T P_{A}=$ 5, $F P_{A}=5, T P_{B}=10$, and $F P_{B}=30$. Table 1 shows the proper interpolation of the intermediate points between $A$ and $B$, with the local skew of 5 negatives for
Table 1. Correct interpolation between two points in PR space for a dataset with 20 positive and 2000 negative examples

\begin{tabular}{|c|c|c|c|c|}
\hline & TP & FP & REC & PREC \\
\hline \hline $\mathrm{A}$ & 5 & 5 & 0.25 & 0.500 \\
. & 6 & 10 & 0.30 & 0.375 \\
. & 7 & 15 & 0.35 & 0.318 \\
. & 8 & 20 & 0.40 & 0.286 \\
. & 9 & 25 & 0.45 & 0.265 \\
$\mathrm{~B}$ & 10 & 30 & 0.50 & 0.250 \\
\hline
\end{tabular}

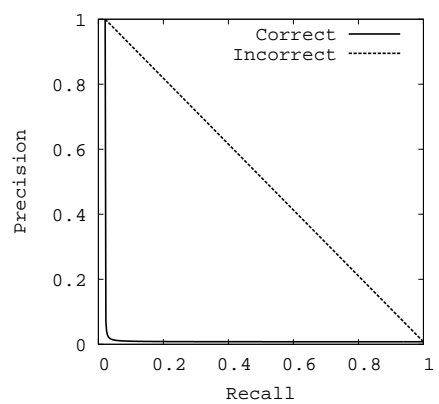

Figure 6. The effect of incorrect interpolation in PR space

every 1 positive. Notice how the resulting Precision interpolation is not linear between 0.50 and 0.25 .

Often, the area under the curve is used as a simple metric to define how an algorithm performs over the whole space (Bradley, 1997; Davis et al., 2005; Goadrich et al., 2004; Kok \& Domingos, 2005; Macskassy \& Provost, 2005; Singla \& Domingos, 2005). The area under the ROC curve (AUC-ROC) can be calculated by using the trapezoidal areas created between each ROC point, and is equivalent to the Wilcoxon-MannWhitney statistic (Cortes \& Mohri, 2003). By including our intermediate $\mathrm{PR}$ points, we can now use the composite trapezoidal method to approximate the area under the PR curve (AUC-PR).

The effect of incorrect interpolation on the AUC-PR is especially pronounced when two points are far away in Recall and Precision and the local skew is high. Consider a curve (Figure 6) constructed from a single point of $(0.02,1)$, and extended to the endpoints of $(0,1)$ and $(1,0.008)$ as described above (for this example, our dataset contains 433 positives and 56,164 negatives). Interpolating as we have described would have an AUC-PR of 0.031; a linear connection would severely overestimate with an AUC-PR of 0.50.

Now that we have developed interpolation for PR space, we can give the complete algorithm for find- 


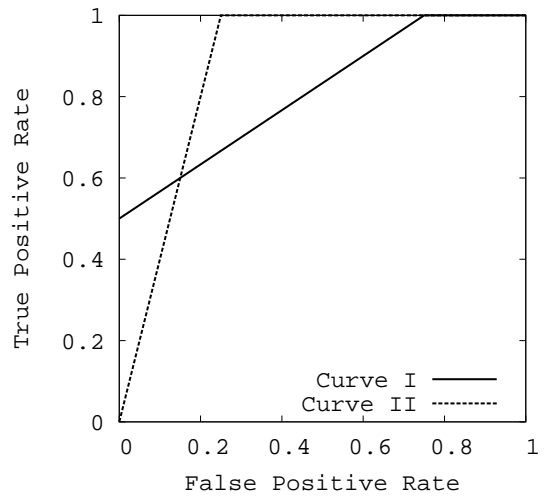

(a) Comparing AUC-ROC for two algorithms

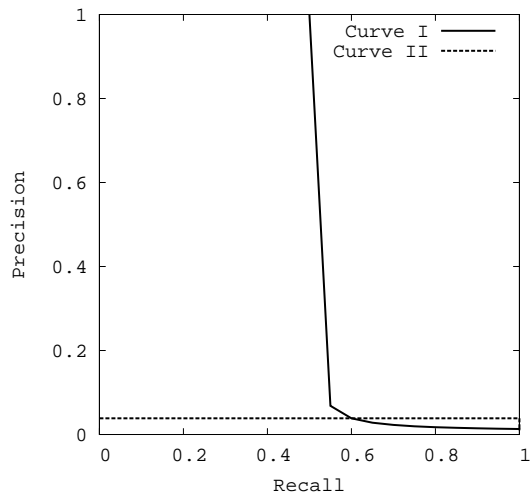

(b) Comparing AUC-PR for two algorithms

Figure 7. Difference in optimizing area under the curve in each space

ing the achievable PR curve. First, we find the convex hull in ROC space (Corollary 3.1). Next, for each point selected by the algorithm to be included in the hull, we use the confusion matrix that defines that point to construct the corresponding point in PR space (Theorem 3.1). Finally, we perform the correct interpolation between the newly created PR points.

\section{Optimizing Area Under the Curve.}

Several researchers have investigated using AUC-ROC to inform the search heuristics of their algorithms. Ferri et al. (2002) alter decision trees to use the AUC-ROC as their splitting criterion, Cortes and Mohri (2003) show that the boosting algorithm RankBoost (Freund et al., 1998) is also well-suited to optimize the AUC-ROC, Joachims (2005) presents a generalization of Support Vector Machines which can optimize AUC-ROC among other ranking metrics, Prati and Flach (2005) use a rule selection algorithm to directly create the convex hull in ROC space, and both Yan et al. (2003) and Herschtal and Raskutti (2004) explore ways to optimize the AUC-ROC within neural networks. Also, ILP algorithms such as Aleph (Srinivasan, 2003) can be changed to use heuristics related to $\mathrm{ROC}$ or PR space, at least in relation to an individual rule.

Knowing that a convex hull in ROC space can be translated into the achievable curve in Precision-Recall space leads to another open question: do algorithms which optimize the AUC-ROC also optimize the AUCPR? Unfortunately, the answer generally is no, and we prove this by the following counter-example. Figure 7 (a) shows two overlapping curves in ROC space for a domain with 20 positive examples and 2000 neg- ative examples, where each curve individually is a convex hull. The AUC-ROC for curve $\mathrm{I}$ is 0.813 and the AUC-ROC for curve II is 0.875 , so an algorithm optimizing the AUC-ROC and choosing between these two rankings would choose curve II. However, Figure 7(b) shows the same curves translated into PR space, and the difference here is drastic. The AUC-PR for curve $\mathrm{I}$ is now 0.514 due to the high ranking of over half of the positive examples, while the AUC-PR for curve II is far less at 0.038 , so the direct opposite choice of curve I should be made to optimize the AUC-PR. This is because in PR space the main contribution comes from achieving a lower Recall range with higher Precision. Nevertheless, based on Theorem 3.2 ROC curves are useful in an algorithm that optimizes AUC-PR. An algorithm can find the convex hull in ROC space, convert that curve to $\mathrm{PR}$ space for an achievable PR curve, and score the classifier by the area under this achievable PR curve.

\section{Conclusions}

This work makes four important contributions. First, for any dataset, the ROC curve and PR curve for a given algorithm contain the same points. This equivalence, leads to the surprising theorem that a curve dominates in ROC space if and only if it dominates in PR space. Second, as a corollary to the theorem we show the existence of the PR space analog to the convex hull in ROC space, which we call an achievable PR curve. Remarkably, when constructing the achievable PR curve one discards exactly the same points omitted by the convex hull in ROC space. Consequently, we can efficiently compute the achievable PR curve. Third, we show that simple linear interpolation is insufficient between points in PR space. Finally, we show 
that an algorithm that optimizes the area under the ROC curve is not guaranteed to optimize the area under the PR curve.

\section{Acknowledgements}

A Java program for calculating all of the discussed metrics can be found at http://www.cs.wisc.edu/ ${ }^{\text {richm/programs/AUC/. }}$

We gratefully acknowledge the funding from USA NLM Grant 5T15LM007359-02 and USA Air Force Grant F30602-01-2-0571, Vítor Santos Costa, Louis Oliphant, our advisors David Page and Jude Shavlik and our anonymous reviewers for their helpful comments and suggestions.

\section{References}

Bockhorst, J., \& Craven, M. (2005). Markov networks for detecting overlapping elements in sequence data. Neural Information Processing Systems 17 (NIPS). MIT Press.

Bradley, A. (1997). The use of the area under the ROC curve in the evaluation of machine learning algorithms. Pattern Recognition, 30, 1145-1159.

Bunescu, R., Ge, R., Kate, R., Marcotte, E., Mooney, R., Ramani, A., \& Wong, Y. (2004). Comparative Experiments on Learning Information Extractors for Proteins and their Interactions. Journal of Artificial Intelligence in Medicine, 139-155.

Cormen, T. H., Leiserson, Charles, E., \& Rivest, R. L. (1990). Introduction to algorithms. MIT Press.

Cortes, C., \& Mohri, M. (2003). AUC optimization vs. error rate minimization. Neural Information Processing Systems 15 (NIPS). MIT Press.

Davis, J., Burnside, E., Dutra, I., Page, D., Ramakrishnan, R., Costa, V. S., \& Shavlik, J. (2005). View learning for statistical relational learning: With an application to mammography. Proceeding of the 19th International Joint Conference on Artificial Intelligence. Edinburgh, Scotland.

Drummond, C., \& Holte, R. (2000). Explicitly representing expected cost: an alternative to $\mathrm{ROC}$ representation. Proceeding of Knowledge Discovery and Datamining (pp. 198-207).

Drummond, C., \& Holte, R. C. (2004). What ROC curves can't do (and cost curves can). ROCAI (pp. 19-26).

Ferri, C., Flach, P., \& Henrandez-Orallo, J. (2002). Learning decision trees using area under the ROC curve. Proceedings of the 19th International Conference on Machine Learning (pp. 139-146). Morgan Kaufmann.

Freund, Y., Iyer, R., Schapire, R., \& Singer, Y. (1998). An efficient boosting algorithm for combining preferences. Proceedings of the 15th International Conference on Machine Learning (pp. 170-178). Madison, US: Morgan Kaufmann Publishers, San Francisco, US.
Goadrich, M., Oliphant, L., \& Shavlik, J. (2004). Learning ensembles of first-order clauses for recall-precision curves: A case study in biomedical information extraction. Proceedings of the 14th International Conference on Inductive Logic Programming (ILP). Porto, Portugal.

Herschtal, A., \& Raskutti, B. (2004). Optimising area under the ROC curve using gradient descent. Proceedings of the 21st International Conference on Machine Learning (p. 49). New York, NY, USA: ACM Press.

Joachims, T. (2005). A support vector method for multivariate performance measures. Proceedings of the 22nd International Conference on Machine Learning. ACM Press.

Kok, S., \& Domingos, P. (2005). Learning the structure of Markov Logic Networks. Proceedings of 22nd International Conference on Machine Learning (pp. 441-448). ACM Press.

Macskassy, S., \& Provost, F. (2005). Suspicion scoring based on guilt-by-association, collective inference, and focused data access. International Conference on Intelligence Analysis.

Manning, C., \& Schutze, H. (1999). Foundations of statistical natural language processing. MIT Press.

Prati, R., \& Flach, P. (2005). ROCCER: an algorithm for rule learning based on ROC analysis. Proceeding of the 19th International Joint Conference on Artificial Intelligence. Edinburgh, Scotland.

Provost, F., Fawcett, T., \& Kohavi, R. (1998). The case against accuracy estimation for comparing induction algorithms. Proceeding of the 15th International Conference on Machine Learning (pp. 445-453). Morgan Kaufmann, San Francisco, CA.

Raghavan, V., Bollmann, P., \& Jung, G. S. (1989). A critical investigation of recall and precision as measures of retrieval system performance. ACM Trans. Inf. Syst., 7, 205-229.

Singla, P., \& Domingos, P. (2005). Discriminative training of Markov Logic Networks. Proceedings of the 20th $\mathrm{Na}$ tional Conference on Artificial Intelligene (AAAI) (pp. 868-873). AAAI Press.

Srinivasan, A. (2003). The Aleph Manual Version 4. http://web.comlab.ox.ac.uk/ oucl/ research/areas/ machlearn/Aleph/.

Yan, L., Dodier, R., Mozer, M., \& Wolniewicz, R. (2003). Optimizing classifier performance via the WilcoxonMann-Whitney statistics. Proceedings of the 20th International Conference on Machine Learning. 